\title{
Vergleichsuntersuchungen zur TSH-Stimulation nach intravenöser und oraler TRH-Gabe
}

\author{
P. Bottermann, U. Henderkott, C. Glogger und H. Gyaram \\ II. Medizinische Klinik und Poliklinik der Technischen Universität München (Direktor Prof. Dr. med. H. Ley) - Klinikum rechts der Isar -
}

\section{Zusammenfassung:}

Bei schilddrüsengesunden Personen. Patienten mit euthyreoter Struma sowie Patienten mit klinisch manifester Hypothyreose und klinisch manifester Hyperthyreose wurde das Ausmaß des TSH-Anstieges nach intravenöser und oraler TRH-Stimulation untersucht. Nach oraler TRH-Gabe kam es bei den drei erstgenannten Gruppen regelhaft zu einem höheren Anstieg der TSH-Konzentration als nach intravenöser TRH-Gabe. Bei manifest hyperthyreoten Patienten fehlte dagegen der TSH-Anstieg sowohl nach intravenöser als auch nach oraler Stimulation. Bei Patienten mit Schilddrüsenhormonkonzentrationen im Normbereich, aber "negativem“ TRH-Test nach intravenöser TRH-Gabe konnte in knapp einem Fünttel der Fälle durch orale TRH-Gabe ein normaler TSH-Anstieg erzielt werden. Von einer .,subklinischen" Hyperthyreose sollte daher erst gesprochen werden, wenn auch der orale TRH-Stimulationstest negativ ausfällt.

\section{Schlüsse/wörter:}

i.v. TRH-Test - oraler TRH-Test - TSH-Stimulierbarkeit - präklinische Hyperthyreose

\begin{abstract}
Summary:
The degree of TSH response after intravenous and oral TRH stimulation was investigated in euthyroid persons, in patients with euthyroid goitre as well as in patients with clinical manifestation of hypothyroidism and hyperthyroidism. In the first three groups mentioned a higher increase of the TSH concentration was observed after oral than after intravenous TRH application. In the case of clinically overt hyperthyroidism TSH increase was absent after both intravenous and oral stimulation. In patients with thyroid hormones concentrations in the normal range, but with "negative" TRH test after intravenous TRH stimulation, a normal TSH increase could be achieved in almost one fifth of the cases after oral TRH stimulation. Consequently, the denotation "subclinical" hyperthyroidism is relevant only if the oral TRH stimulation, too, failed.
\end{abstract}

Keywords:

i.v. TRH test --oral TRH test - TSH stimulation - preclinical hyperthyroidism

\section{Einleitung}

Der Thyreotropin-Releasing-Hormon (TRH)-Stimulationstest hat sich in der Diagnostik von Schilddrüsenfunktionsstörungen seit Jahren bewährt (1-3). Die TRH-Gabe kann sowohl intravenös als auch oral erfolgen (4). Von den meisten Untersuchern wird der i.v. TRH-Test mit Gabe von 200 oder $400 \mu \mathrm{g}$ TRH bevorzugt. Der orale TRH-Test mit Gabe von $40 \mathrm{mg}$ TRH gilt jedoch als stimulationsstärker $(4,5-8,10$, 12).

Ein stärkerer Stimulationseffekt könnte in Grenzfällen zur besseren Differenzierung zwischen euthyreoter und hyperthyreoter Stoffwechsellage beitragen $(1,2,6,8,9)$. Deswegen wurde die TSH-Antwort nach intravenöser und oraler TRHGabe in einer ersten Untersuchungsserie zunächst bei gesunden Probanden. Struma-Patienten und Patienten mit eindeutigen Schilddrüsenfunktionsstörungen wie manifester Hypothyreose und manifester Hyperthyreose verglichen. In einer zweiten Untersuchungsserie wurde dann der Frage einer „unzureichenden" TSH-Antwort bei Patienten mit fraglich hyperthyreoter Regelkreisstörung durch Vergleich der TSHAntwort nach intravenöser und oraler TRH-Gabe nachgegangen.

\section{Versuchsanordnung und Methodik}

In der ersten Untersuchungsserie wurden 15 freiwillige stoffwechselgesunde Personen ohne Schilddrüsenveränderungen, 15 Patienten mit sog. euthyreoter blander Struma II, sieben Patienten mit klinisch manifester Hypothyreose und 14 Patienten mit klinisch manifester Hyperthyreose (Immunhyperthyreose vom Basedow-Typ oder umschriebene thyreoidale Autonomie) untersucht. Die Diagnosen waren durch Bestimmung des Gesamtthyroxins $\left(T_{4}-D\right)$, der freien Schilddrüsenhormonbindungskapazität $\left(T_{3}-U\right)$, Berechnung des 


$$
\begin{aligned}
& \text { Euthyreole Probanisen narth Eulhyrpolo Probanden neteh }
\end{aligned}
$$

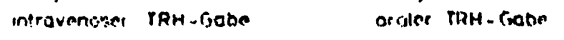

$$
\begin{aligned}
& \text { i. SEM, } n=15 \\
& \text { \& SEM. } n=15
\end{aligned}
$$

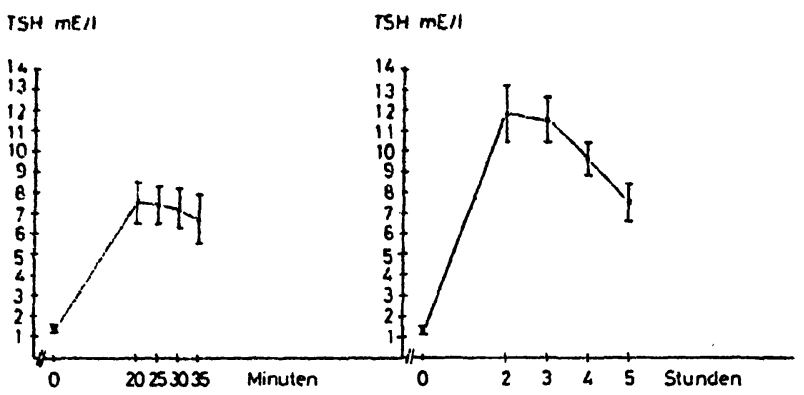

Abb. 1: Verhalten der TSH-Sekretion nach intravenöser $(200 \mu \mathrm{g})$ und oraler $(40 \mathrm{mg}) \mathrm{TRH}-$ Gabe bei 15 schilddrüsengesunden Normalpersonen.

freien Thyroxinindex $\left(\mathrm{FT}_{4}\right)$ und Bestimmung des Gesamttrijodthyronins $\left(T_{3}-R \mid A\right)$ gesichert. Die Annahme einer thyreoidalen Autonomie erfolgte bei szintigrafischem Vorliegen eines dekompensierten autonomen Adenoms. Bei allen Personen wurde nach nächtlicher Nahrungskarenz morgens nüchtern zunächst ein i.v. TRH-Test mit $200 \mu \mathrm{g}$ TRH, zwei bis fünf Tage später ein oraler TRH-Test mit $40 \mathrm{mg}$ TRH durchgeführt. Venöse Blutabnahmen zur TSH-Bestimmung erfolgten vor sowie $20,25,30$ und $35 \mathrm{~min}$ nach i.v. TRH-Gabe und 60, 120 , 180,240 und $300 \mathrm{~min}$ nach oraler TRH-Gabe. Während des i.v. TRH-Testes wurde Nahrungskarenz eingehalten. Dagegen durfte eine Stunde nach oraler TRH-Einnahme gefrühstückt werden.

In die zweite Untersuchungsserie wurden Patienten aufgenommen, bei denen der klinische Verdacht einer Hyperthyreose bestand, die aber normale Schilddrüsenhormonwerte aufwiesen und bei denen gleichzeitig ein ,unzureichender" TSHAnstieg ( $\triangle \mathrm{TSH} 0,55-2,0 \mathrm{mE} / \mathrm{l}) \mathrm{im}$ i.v. TRH-Tést vorlag. Bei
54 von 205 Patienten, die diese Konstellation aufwiesen, war es möglich, ein bis zwei Wochen später erneut einen i.y. TRH. Test durchzuführen und an diesen zweiten i.v. TRH-Test nach 24 Stunden einen oralen TRH-Test anzuschließen. Bei diesel Untersuchungsserie wurde der Stimulationswert nach i.v. TRH-Gabe nach $25 \mathrm{~min}$, nach oraler TRH - Gabe nach $180 \mathrm{~min}$ abgenommen.

Die TSH-Bestimmung erfolgte mittels einer laboreigenen Doppelantikörpermethode, deren untere Nachweisgrenze bei $0,37 \pm 0.11 \mathrm{mE} / \mathrm{l}$ liegt $(\bar{x} \pm S . D$.; $n=55$. Untere Nachweisgrenze festgelegt beim dreifachen $V_{k}$ des $B_{0}$-Wertes.) Es wurden Dreifachbestimmungen durchgeführt. Auf Grund deı (Un)genauigkeit der Bestimmungsmethodik kann ein signifikanter TSH-Anstieg erst dann angenommen werden, wenn die Differenz zwischen Ausgangswert und Stimulationswer $0,55 \mathrm{mE} / \mathrm{l}$ erreicht $(p<0,05)$.

\section{Ergebnisse}

Bei den euthyreoten Probanden (Abbildung 1) wird 20 bis 30 min nach intravenöser TRH-Gabe der höchste TSH-Werl erreicht. Nach oraler TRH-Gabe kommt es zu einem insgesam1 höheren TSH-Anstieg als nach intravenöser TRH-Applikation. Das TSH-Maximum ist nach $120-180 \mathrm{~min} z \mathrm{zu}$ sehen. Nach 240 min fällt der TSH-Spiegel bereits wieder ab.

Ähnliche Verhältnisse bestehen bei den Patienten mit blande। Struma (Abbildung 2). Nach i.v. TRH-Gabe wird das Maximum der TSH-Konzentration ebenfalls zwischen der 20. und 30. Minute beobachtet. Nach oraler TRH-Gabe ist das TSHMaximum wiederum nach 120-180 min zu erkennen. Nact 240 min fällt der TSH-Spiegel auch bei dieser Personengruppe bereits wieder ab. Ebenso wie bei den gesunden Probanden steigt auch hier die TSH-Konzentration nach oraler TRHApplikation höher an als nach intravenöser TRH-Gabe. Dabe werden bei den Struma-Patienten sowohl nach intravenöse। als auch nach oraler TRH-Gabe jeweils etwas höhere TSH. Werte erreicht als bei den gesunden Probanden.

Bei den hypothyreoten Patienten (Abbildung 3 ) waren bereits die TSH-Ausgangswerte deutlich erhöht. Bei stärkereI

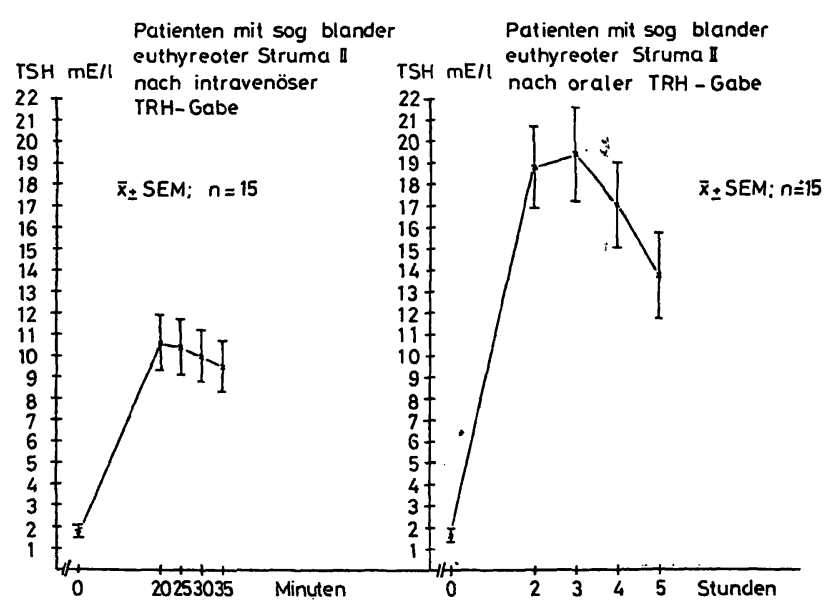

Abb. 2: Verhalten der TSH-Sekretion nach intravenöser $(200 \mu \mathrm{g})$ und oraler (40 mg) TRH-Gabe bei 15 Patienten mit sog. blander euthyreoter Struma II.

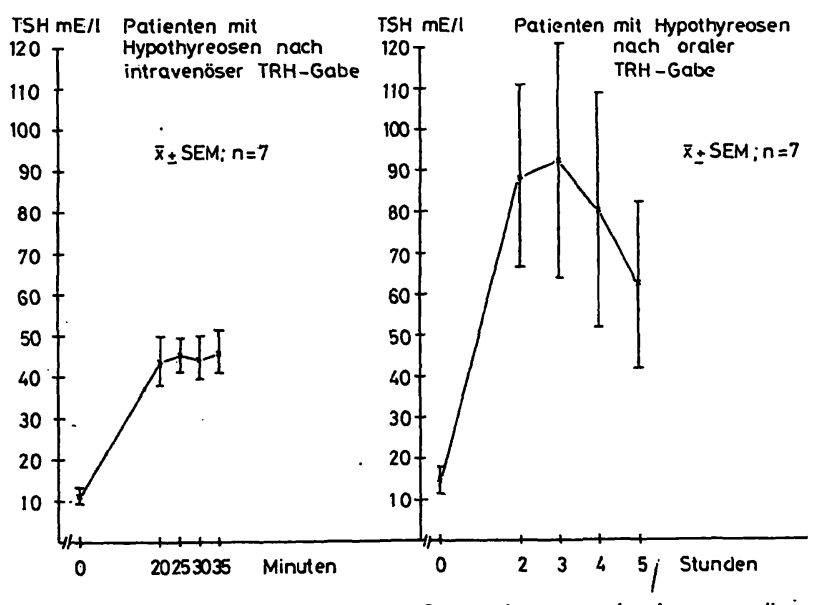

Abb.3: Verhalten der TSH-Sekretion nach intravenösè $(200 \mu \mathrm{g})$ und oraler $(40 \mathrm{mg}) \mathrm{TRH}-\mathrm{Gabe}$ bei sieben Patienten mit manifester Hypothyreose. (Man beachte den im Vergleich zu Abb. 1 und 2 geänderten Maßstab der Ordinate). 
Streuung der Einzelwerte kam es bei allen Patienten zu einem erheblichen TSH-Anstieg. Auch bei dieser Patientengruppe wurden die höchsten TSH-Werte zwischen 20 und $30 \mathrm{~min}$ nach intravenöser TRH-Gabe gesehen. Lediglich bei zwei Patienten wurde das Maximum erst zur 35. Minute erreicht. Nach oraler TRH-Gabe wurden die höchsten TSH-Werte im Mittel nach $180 \mathrm{~min}$ beobachtet. Nach $240 \mathrm{~min}$ war auch bei diesen Patienten bereits ein Wiederabfall der TSH-Konzentration zu erkennen.

Bei den Patienten mit klinisch manifester Hyperthyreose kam es nach intravenöser TRH-Gabe zu keinem signifikanten TSHAnstieg über den Ausgangswert $(\triangle \mathrm{TSH}<0,55 \mathrm{mE} / \mathrm{I})$. Beim oralen TRH-Test wurde bei zwei Patienten ein minimaler TSH-Anstieg $(\triangle T S H)$ von 0,6 und $0,7 \mathrm{mE} / /$ gefunden.

Diese Beobachtung gab letztlich Anstoß zur Durchführung der zweiten Untersuchungsserie, bei der bei Patienten mit „unzureichender" TSH-Stimulierbarkeit im. i.v. TRH-Test ( $\triangle T S H$ 0,55-2,0 mE/I) das Ausmaß der Stimulierbarkeit nach intravenöser und oraler TRH-Gabe miteinander verglichen wurde (Tabelle 1).

Bei sieben der 54 Patienten, bei denen ein zweiter i.v. TRHTest innerhalb von ein bis zwei Wochen durchgeführt werden konnte, kam es beim zweiten i.v. TRH-Test zu einem deutlichen TSH-Anstieg $(\Delta \mathrm{TSH}>2,0 \mathrm{mE} / \mathrm{l})$. Diese Patienten wurden von der weiteren Auswertung ausgeschlossen.

Zehn der verbleibenden 47 Patienten zeigten beim zweiten i.v. TRH-Test einen TSH-Anstieg über 1,0 mE/l, nicht aber über 2,0 mE/I. Nach oraler TRH-Gabe kam es bei diesen Patienten mit einer Ausnahme (1,4 mE/I) immer zu einem TSH-Anstieg über $2.0 \mathrm{mE} / \mathrm{l}$.

Bei elf Patienten mit einem TSH-Anstieg von 0,55-1,0 mE/I im i.v. TRH-Test kam es nach oraler TRH-Gabe bei sechs Patienten zu einem TSH-Anstieg von mehr als $1,0 \mathrm{mE} / \mathrm{I}$ ( $\triangle \mathrm{TSH}>1,0-2,0 \mathrm{mE} / \mathrm{l}$ ), bei drei Patienten zu einem TSHAnstieg zwischen 0,55 und $1,0 \mathrm{mE} / \mathrm{l}$ und bei zwei Patienten zu keinem signifikanten TSH-Anstieg $(\triangle T S H<0,55 \mathrm{mE} / \mathrm{l})$.

Bei 26 Patienten fand sich beim zweiten i.v. TRH-Test kein signifikanter TSH-Anstieg $(\triangle \mathrm{TSH}<0,55 \mathrm{mE} / \mathrm{I})$. Bei 19 von diesen 26 Patienten war nach oraler TRH-Gabe ebenfalls kein signifikanter TSH-Anstieg zU sehen. Bei einem Patienten betrüg der TSH-Anstieg $0.7 \mathrm{mE} / \mathrm{l}$, bei den restlichen sechs Patienten lag er zwischen 1 und $2 \mathrm{mE} / \mathrm{l}$.

\section{Diskussion}

Zur Beurteilung der Schilddrüsenfunktion hat sich der intravenöse TRH-Test mit Bestimmung der TSH-Konzentration vor und 20 bis $30 \mathrm{~min}$ nach TRH-Gabe seit Jahren bewährt (1 4). Die vorliegenden Untersuchungsergebnisse zeigen in Übereinstimmung mit der Literatur $(4-6,8,10-12)$, daß auch mit oraler TRH-Gabe eine zuverlässige Stimulation der TSH-Sekretion erreicht werden kann.

Generell fällt auf, daß die TSH-Konzentration nach oraler TRH-Gabe höher ansteigt als nach intravenöser Applikation. Die TSH-Werte lagen bei den schilddrüsengesunden Probanden und den Patienten mit blander Struma nach oraler Stimulation ca. $11 / 2$ fach höher als nach intravenöser Stimulation. Für den.i.v. TRH-Test ermittelte obere Normwerte können daher nicht direkt auf den oralen TRH-Test übertragen werden, wenn zwischen euthyreoter und hypothyreoter Funktionslage unterschieden werden soll.

Die stärkere Stimulationswirkung der oralen TRH-Gabe dürfte weniger auf die höhere Dosis von $40 \mathrm{mg}$ im Vergleich zur $200 \mu \mathrm{g}$ Dosis im i.v. Test zurückzuführen sein, sondern eher einer prolongierten Stimulation entsprechen, die durch eine länger anhaltende Erhöhung des TRH-Spiegels infolge einer kontinuierlichen TRH-Resorption aus dem Verdauungstrakt bedingt ist $(1,6,9)$. Entsprechend ist der TSH-Konzentrationsanstieg nach i.v. TKH-Gabe kurzdauernd und spitzgipflig, nach oraler TRH-Gabe längerdauernd und mehr plateauförmig. Hieraus ergeben sich auch sog. Vor- oder Nachteile der beiden Teste. Beim i.v. TRH-Test muß die zweite Blutabnahme zeitlich exakt erfolgen, damit das Maximum des TSHAnstieges nicht verpaßt wird. Bei oraler Stimulation sind kurze zeitliche Verschiebungen der zweiten Blutabnahme wegen des plateauartigen TSH-Maximums weniger kritisch. Der i.v. $\mathrm{TRH}$-Test ist rasch beendet, der orale TRH-Test benötigt mehrere Stunden Zeit. Bei einer Entscheidung für oder wider intravenösen oder oralen TRH-Stimulationstest dürften für den Untersucher daher im wesentlichen organisatorische Gesichtspunkte im Vordergrund stehen.

Hinsichtlich der diagnostischen Aussage, ob eine euthyreote oder hypothyreote Funktionslage besteht, dürften beide Teste gleichwertig sein. Differenzierter muß vorgegangen werden, wenn eine hyperthyreote Funktionslage zur Diskussion steht. Bei ausreichendem TSH-Anstieg (,,positiver" TRH-Test) läßt sich eine Hyperthyreose sowohl mit dem intravenösen als auch dem oralen TRH-Test ausschließen. Wir sehen in Übereinstimmung mit den meisten Untersuchern einen TSHAnstieg von mehr als 2,0 mE/l ( $\triangle \mathrm{TSH}>2,0 \mathrm{mE} / \mathrm{l})$ als ausreichend an $(1-3)$. Fehlt der TSH-Anstieg bei gleichzeitig erhöhten Schilddrüsenhormonkonzentrationen, kann von einer hyperthyreoten Stoffwechselsituation ausgegangen werden.

Schwierigkeiten bereitet dagegen die Beurteilung des Schilddrüsenfunktionszustandes, wenn die Schilddrüsenhormonkonzentration im oberen Normbereich liegt, gleichzeitig aber der intravenöse TRH-Test „,negativ" ausfällt, d.h. keine (,no response") oder nur eine geringfügige (,low response") TSH-Stimulierbarkeit nach intravenöser TRH-Gabe besteht $(1,2,6)$. Für diese Konstellation wurde der Begriff der "subklinischen” oder „,präklinischen“ Hyperthyreose geprägt $(1,9,13)$. Jedoch läßt sich bei einem Teil dieser Patienten mit oraler TRH-Gabe noch ein deutlicher TSH-Anstieg erzielen, so daß bei diesen Patienten offenbar noch keine hyperthyreote Regelkreisstörung vorzuliegen scheint $(1,2,5,6,9,11,12)$.

Wir fanden bei 205 Patienten mit normalen Schilddrüsenhormonkonzentrationen im i.v. TRH-Test einen unzureichenden TSH-Anstieg ( $\triangle T S H$ 0,55-2,0 mE/I). Bei diesen Patienten war daher ein oraler TRH-Test indiziert.

Im Gegensatz zur ersten Untersuchungsserie schien es sinnvoll, den oralen TRH-Test nicht mit dem mehrere Tage zuvor durchgeführten intravenösen TRH-Test zu vergleichen, sondern vielmehr zunächst den i.v. TRH-Test zu wiederholen, um 24 Stunden später den oralen TRH-Test anzuschließen. Durch dieses Vorgehen sollte ein möglichst geringer zeitlicher $A b$ stand zwischen intravenöser und oraler TRH-Belastung gewährleistet werden. Da von einer grenzwertig hyperthyreoten/euthyreoten Schilddrüsenfunktionslage auszugehen war, schien es ungünstig. Ergebnisse von Untersuchungen zu 
vergleichen, zwischen denen ein mehrtagiger Abstand lag Mogliche Fehlinterpretationen durch zwischenzeitliche Anderungen der grenzwertigen Schilddrusenfunktionslago wa. ren derart am besten auszuschließen.

Zwerter intravenoser TRH-Test und nachfolgender oraler TRH-Test konnten aus außeren Grunden nur bei 54 der 205 Pationton durchgefuhrt werden. In der Tat fiel bei sieben Patienten der zweite i.v. TRH-Test mit einem TSH-Anstieg von über $2.0 \mathrm{mE} / 1$ normal aus. Es wurden also nur bei den verbleibenden 47 Patienten die Ergebnisse des zweiten i.v. TRH-Testes mit den Ergebnissen des oralen TRH-Testes verglichen. Von diesen 47 Patienten wiesen zehn Patienten mit einem TSH-Anstieg zwischen 1,0 und $2,0 \mathrm{mE} / \mathrm{l}$ nach intravenoser TRH-Gabe - also unzureichender TSH-Antwort - eine normale Stimulierbarkeit mit einem TSH-Anstieg über 2.0 mE/I im oralen TRH-Test auf (Tabelle). Diese Patienten waren also nicht hyperthyreot. Bei 26 Patienten mit fehlender TSH-Antwort ( $\triangle T S H<0,55 \mathrm{mE} / \mathrm{I})$ im i.v. TRH-Test war bei 19 Patienten durch orale TRH-Gabe ebenfalls keine TSHStinulation zu erzielen. Bei den anderen sieben Patienten sowie den restlichen elf Patienten blieb die TSH-Antwort mit zwei Ausnahmen auch nach oraler Stimulation unzureichend ( $\triangle T S H$ 0,55-2,0 mE/I). Weitere Untergliederungen der Unrersuchungsergebnisse sind der Tabelle zu entnehmen.

Tab. 1: TSH-Verhalten nach intravenöser und oraler TRHGabe bei Patienten, bei denen zuvor im intravenösen TRH. Test ein „unzureichender" TSH-Anstieg (.,low response": $\triangle T S H$ 0.55-2,0 mE/I) festgestellt wurde.

\begin{tabular}{|c|c|c|c|}
\hline \multicolumn{2}{|c|}{$\begin{array}{l}\text { Zweiter intravenöser } \\
\text { TRH-Test }\end{array}$} & \multicolumn{2}{|c|}{ Oraler TRH-Test } \\
\hline$N$ & $\mathrm{TSH}(\mathrm{mE} / \mathrm{l})$ & $\mathrm{TSH}(\mathrm{mE} / \mathrm{l})$ & $N$ \\
\hline 7 & $>2,0$ & $>2,0$ & 7 \\
\hline 10 & $>1,0-2,0$ & $\begin{array}{l}>2,0 \\
>1,0-2,0\end{array}$ & $\begin{array}{l}9 \\
1\end{array}$ \\
\hline 11 & $0,55-1,0$ & $\begin{array}{l}>1,0-2,0 \quad \\
0,55-1,0 \\
<0,55\end{array}$ & $\begin{array}{l}6 \\
3 \\
2 \\
\end{array}$ \\
\hline 26 & $<0,55$ & $\begin{array}{l}>1,0-2,0 \\
0,55-1,0 \\
<0,55\end{array}$ & $\begin{array}{r}6 \\
1 \\
19\end{array}$ \\
\hline
\end{tabular}

Mit Hilfe des stimulationsstärkeren oralen TRH-Testes gelang es also, bei neun von 47 Patienten - das sind ca. $19 \%$ - eine Hyperthyreose bzw. eine hyperthyreote Regelkreisstörung auszuschließen. Von anderen Untersuchern wurde bei allerdings anderen Grenzkriterien sogar bei 24 von 42 Patienten, die im i.v. TRH-Test keinen oder einen subnormalen TSHAnstieg zeigten, über eine normale TSH-Antwort näch oraler TRH-Gabe (5) sowie von einer Abnahme negativer TRHTeste von $12 \%$ auf $4,5 \%$ bei Übergang von intravenöser auf orale TRH-Stimulation (6) berichtet.

Bei normaler Schilddrüsenhormonkonzentration und gleichzeitig unzureichendem TSH-Anstieg (,low response") im i.v. TRH-Test sollte daher ein oraler TRH-Stimulationstest durchgeführt werden, bevor die .Diagnose einer "subklinischen" oder „präklinischen” Hyperthyreose gestellt wird. insgesamt können intravenöser und oraler TRH-Stimulationstest in der Schilddrüsenfunktionsdiagnostik als etwa gleich. wertig bezeichnet werden. Mit beiden Testverfahren lassen sich Funktionsstorungen gut erfassen. In Grenzfällen mit fraglich euthyreoter/hyperthyreoter Regelkreisfunktion bietet der stimulationsstärkere orale TRH-Test gewisse Vorteile. da die Zahl „negativer" Testausfälle etwas niedriger liegt.

Die TRH-Teste wurden mit "Thyroliberin" (Merck) und "Antepan" (Henning, Berlin) durchgeführt. Wir danken den Firmen für die Uberlassung der Praparate.

\section{Schriftum:}

1. STAUB, J. J. Der TRH-Test: Seine Bedeurung für die Praxis. Schwerz. Rundschau Med (Praxis) 66, 33-36 (1977).

2. WENZEL, K.-W.: Der TRH-Test zur rationellen und rationalen Schilddrusendiagnostik Disch. Med. Wschr. 104. $229-234$ (1979)

3. PFANNENSTIEL, P., BORNER, W.. DROESE, M., EMRICH. D., ERHARDT, F., HACKENBERG, K. HEINZE, H. G., HERRMANN, J., HESCH, R. D., HORN, K., HORSTER, F. A. JOSEPH, K., KLEIN, E., KRÚSKEMPER, H. L.. VON ZUR MUUHLEN, A., OBERHAUSEN. E., REINWEIN, D., RUDORFF, K. H., SCHATZ, H., SCHLEUSENER, H.. SCRIBA. P. C.. WENZEL, K. W.: Methoden und ihr stufenweiser Einsatz bei der Diagnostik von Schilddrüsenerkrankungen. Empfehlungen der Sektion Schilddrüse der Deutschen Gesellschaft fur Endokrinologie. Internist. Welt 2. 99-106 (1979).

4. BOTTERMANN, P.. GLOGGER. G., HENDERKOTT, U.: Intravenöser und oraler TRHStimulationstest. Med. Klin. 74, 1485-1491 (1979).

5. JÜNGST, D., SCHINEIS, E., VOGT, W.. BÜLL, U., MANN, K., KARL, H. J.: Parameter of hyroid function in thyroid autonomy. J. Mol. Med. 4. 103-109 (1980)

. STAUB. J. J., GSAARD, J., GEMSENJAGER. E.: Entwicklung eines einfachen oralen Kurtests mit dem TSH-Releasing-Hormon (TRH) und dessen Anwendung in der Schilddrü7.

7. GEMSENJAGER, E., STAUB, J.J., GIRARD.J.. HEITZ, Ph.: Preclinical Hyperthyroidism in Multinodular Goirer.

GEAREN J., GEMSER, E.: Oral T R.H for

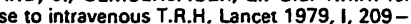
210 .

9. BIRKHÄUSER, M. H., STAUB, J. J., GRÄNI, R., GIRARD, J., BAUR, U., GEMSENJÄGER, E.: Bedeutung des oralen TRH-Tests zur Beurteilung der Schilddrüsenfunktion bei verminderter TSH-Reserve. Schweiz. Med. Wschr. 109, 1898-1900 (1979).

10. STAUB, J. J.: Vorteile des oralen TRH-Tests für die Schilddrüsendiagnostik. Dtsch.

Med. Wschr. 104, 1019 (1979).
11. HOENLE, R.: Mehr diagnostische Sicherheit in der AusschluBdiagnostik der Hyperthy11. HOENLE, R.: Mehr diagnostische Sicherheit in der Ausschlußdiagnostik der Hyperthyreose durch den TRH-Stimulationstest mit Thyroliberin. Vergleichsuntersuchung bei euthyreoten

12. RINCK, P. A., BOTSCH, H., ROSSDEUTSCHER, R.: Zur Indikation des oralen TRHTestes. Fortschr. Röntgenstr. 134, 198-200 (1981).

restes. Fortschr. Rontgenstr. 134, 198-200 (1981). 13. STUDR, H., BUERGI, H., KOENI, M. P.. Die klinische Bedeutung der

\section{Anschrift des Verfassers:}

Prof. Dr. med. P. Bottermann

II. Medizinische Klinik und Poliklinik der TUM

- Klinikum rechts der Isar -

Ismaninger Straße 22

D-8000 München 80 